\title{
聚芳撑乙炔微孔骨架材料的合成及其气体吸附
}

\author{
徐佳伟 ${ }^{a} \quad$ 张崇 $^{b} \quad$ 王迅昶 $^{a} \quad$ 蒋加兴*, 汪锋*, $^{*}$ \\ ( ${ }^{a}$ 武汉工程大学化工与制药学院 武汉 430073) \\ ( ${ }^{b}$ 陕西师范大学材料科学与工程学院 西安 710062)
}

\begin{abstract}
摘要 有机微孔聚合物(MOPs) 在气体存储、吸附分离和非均相催化等领域具有优良性质而广受关注. 近年来, 聚芳撑 乙炔微孔骨架材料的研究成为 MOPs 领域中的热点. 分别以三(4-乙炔基)苯胺、甲基三(4-乙炔基苯基)硅烷、苯基三(4乙炔基苯基)硅烷为基本构筑单元, 通过端炔基氧化均聚的方法, 制备了三种聚芳撑乙炔微孔骨架材料, 研究了结构组 成对制备聚合物孔道性能和气体吸附性能的影响. 氮气吸附测试结果表明, 聚合物的 Brunauer-Emmett-Teller (BET)比 表面积的范围在 $602 \sim 715 \mathrm{~m}^{2} \cdot \mathrm{g}^{-1}$. 由于骨架中含有富氮基团(三苯胺)以及具有较大的比表面积, 在 $1.13 \mathrm{bar} / 273 \mathrm{~K}$ 条件 下, 聚三(4-乙炔基)苯胺(TEPA-MOP)的 $\mathrm{CO}_{2}$ 吸附能力为 $1.59 \mathrm{mmol} \cdot \mathrm{g}^{-1}$. 此外, TEPA-MOP 和聚苯基三(4-乙炔基苯基)硅 烷(TEPP-MOP)具有优良的选择性吸附性能, 对 $\mathrm{CO}_{2} / \mathrm{N}_{2}$ 的选择性吸附分别是 69.9 和 73.2. 聚合物 TEPA-MOP 具有优异 的 $\mathrm{CO}_{2} / \mathrm{N}_{2}$ 的选择吸附性和适中的 $\mathrm{CO}_{2}$ 吸附能力, 因此将在气体吸附与分离方面具有潜在的应用前景. 关键词 有机微孔聚合物; 聚芳撑乙炔; 氧化均聚; 气体吸附; 分离
\end{abstract}

\section{Synthesis and Gas Sorption Properties of Microporous Poly(arylene ethynylene) Frameworks}

\author{
$\mathrm{Xu}$ Jiawei $^{a} \quad$ Zhang, Chong $^{b} \quad$ Wang, Xunchang ${ }^{a} \quad$ Jiang, Jiaxing*, ${ }^{*} \quad$ Wang, Feng*,a \\ ( ${ }^{a}$ School of Chemical Engineering and Pharmacy, Wuhan Institute of Technology, Wuhan 430073) \\ ( ${ }^{b}$ School of Materials Science and Engineering, Shaanxi Normal University, Xi'an 710062)
}

\begin{abstract}
Microporous organic polymers (MOPs) have drawn much attention because of their potential applications such as gas storage, separation and heterogeneous catalysis. There is great interest in the design, synthesis and property evaluation of poly(arylene ethynylenes) (PAEs) with intrinsic microporosity. In addition to Sonogashira coupling reaction between terminal alkynes and halides, the oxidative dimerization of terminal alkynes is an alternating strategy for the buildup of the microporous PAE frameworks. In this paper, a series of MOPs were synthesized by the oxidative dimerization of terminal alkynes using triethynyl monomers such as tris(4-ethynylphenyl)amine, tris(4-ethynylphenyl)methylsilane and polytris(4-ethynylphenyl)phenylsilane. The resulting MOPs were characterized by FT-IR spectra, thermogravimetric analysis (TGA), scanning electron microscopy (SEM), transmission electron microscopy (TEM), powder X-ray diffraction (PXRD) measurements. FT-IR spectra indicate the success of the homocoupling reaction for constructing the dialkyne-bridged polymer frameworks. These polymer frameworks exhibit high thermal stability with onset of decomposition temperature above $350{ }^{\circ} \mathrm{C}$ at $5 \%$ mass loss under nitrogen flow. PXRD and TEM measurements revealed that all the polymer frameworks are amorphous solid in nature. These dialkyne-bridged MOPs exhibit moderate surface areas ranging from 602 to $715 \mathrm{~m}^{2} \cdot \mathrm{g}^{-1}$. The incorporation of triphenylamine moieties into the polymer skeleton increases the number of electron donating basic nitrogen sites in the porous frameworks. Thus, the triphenylamine-based polymer polytris(4-ethynylphenyl)amine (TEPA-MOP) with the highest Brunauer-Emmett-Teller (BET) surface area shows the highest $\mathrm{CO}_{2}$ uptake capacity of 1.59 mmol $\bullet \mathrm{g}^{-1}$ at $273 \mathrm{~K}$ and 1.13 bar among the resulting polymer frameworks. In addition, TEPA-MOP showed the highest $\mathrm{H}_{2}$ adsorption up to $1.04 \mathrm{wt} \%$ at 1.13 bar and $77 \mathrm{~K}$ and polytris(4-ethynylphenyl)phenylsilane (TEPP-MOP) displayed the lowest $\mathrm{H}_{2}$ adsorption of $0.64 \mathrm{wt} \%$ at the same conditions. As for separation of $\mathrm{CO}_{2}$, both TEPA-MOP and TEPP-MOP exhibit relatively high $\mathrm{CO}_{2}$-over- $\mathrm{N}_{2}$ selectivities of 69.9 and 73.2 at $273 \mathrm{~K}$, respectively. The above results show that TEPA-MOP might be the good candidate for the balanced $\mathrm{CO}_{2}$ uptake capacity with impressive $\mathrm{CO}_{2} / \mathrm{N}_{2}$ selectivity among the microporous PAE frameworks.

Keywords microporous organic polymer; poly(arylene ethynylene); oxidative dimerization; gas adsorption; separation
\end{abstract}

* E-mail: psfwang@wit.edu.cn, jiaxing@snnu.edu.cn

Received February 17, 2017; published April 9, 2017.

Supporting information for this article is available free of charge via the Internet at http:/sioc-journal.cn.

Project supported by the the Natural Science Foundation of China (No. 51103111) and the Education Ministry of China (No. NCET-12-0714).

项目受国家自然科学基金(No. 51103111)和教育部新世纪优秀人才支持计划(No. NCET-12-0714)资助. 


\section{1 引言}

传统化石能源的燃烧释放大量 $\mathrm{CO}_{2}$ 是导致温室效 应和环境污染的主要原因, 因此对烟气中的 $\mathrm{CO}_{2}$ 进行捕 获是减少 $\mathrm{CO}_{2}$ 含量的有效方法 ${ }^{[1]}$. 由于有机胺和 $\mathrm{CaO}$ 吸 附法存在较高的能耗和较大的污染等不足之处, 广大科 研人员尝试开发有效捕获 $\mathrm{CO}_{2}$ 的各种新型材料 ${ }^{[2 \sim 4]}$.

多孔固体如活性炭、无机分子篮、金属有机骨架 $(\mathrm{MOFs})$ 、有机微孔聚合物(MOPs) 等以物理吸附的方式 吸收 $\mathrm{CO}_{2}$ 受到研究人员的极大关注. 其中 MOPs 具有合 成策略多样性、物化性质稳定、比表面积高、骨架密度 低、孔道结构易功能化修饰等特点, 因此在气体吸附领 域有广泛的应用前景 ${ }^{\left[{ }^{[} \sim 7\right]}$. 迄今为止, 已经有一系列的 新型 MOPs 被开发出来, 包括共轭微孔聚合物 $(\mathrm{CMPs})^{[8 \sim 10]}$ 、自具微孔聚合物(PIMs) ${ }^{[11,12]}$ 、共价有机框 架 $(\mathrm{COFs})^{[13 \sim 15]}$ 、超交联聚合物 $(\mathrm{HCPs})^{[16 \sim 18]}$ 、共价三嗪 系框架 $(\mathrm{CTFs})^{[19]}$ 和多孔芳环框架 $(\mathrm{PAFs})^{[20,21]}$.

聚芳撑乙炔(PAE)微孔骨架材料具有微孔率较大、 化学及热稳定性较高和孔径可调等特点, 从而受到广泛

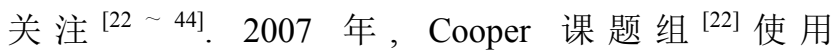
Sonogashira-Hagihara 偶联反应首次制备了微孔结构的 PAE 化合物, 其 Brunauer-Emmett-Teller (BET)比表面积 高达 $834 \mathrm{~m}^{2} \cdot \mathrm{g}^{-1}, \mathrm{H}_{2}$ 的吸附能力为 $1.16 \mathrm{wt} \%(1 \mathrm{bar} / 77 \mathrm{~K})$. 随后, Cooper 课题组 ${ }^{[23 ~ 25]}$ 进行 PAE 微孔骨架材料的系 统研究工作, 制备了一系列比表面积高、气体吸附性能

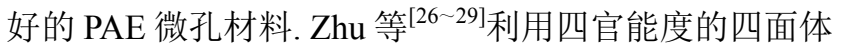
型立体小分子四炔四苯基甲烷单体, 制备系列微孔聚合 物. 由于 $\mathrm{OH}, \mathrm{NH}_{2}$ 等极性基团或金属离子被引入到聚合 物骨架结构中, 该类型微孔聚合物的吸附性能得到了提 高. $\mathrm{Han}$ 等 ${ }^{[35]}$ 和 $\mathrm{Ma}$ 等 ${ }^{[36]}$ 分别合成了四炔螺二荡基微孔 聚合物, 在 $1 \mathrm{bar} / 273 \mathrm{~K}$ 条件下 $\mathrm{CO}_{2}$ 吸附能力为 $7.88 \sim$ $12.01 \mathrm{wt} \%$. 含氮结构可提高对 $\mathrm{CO}_{2}$ 酸性气体的吸附能 力. 磷与氮是同族相邻元素, 具有相同的最外层电子数. Yang 课题组 ${ }^{[37,38}$ 报道了系列三炔三苯基磷基微孔聚合 物, 得到的富磷微孔骨架对 $\mathrm{CO}_{2}$ 表现出较好的吸附性 能. Zhang 等 ${ }^{\left[{ }^{39]}\right.}$ 采用微乳液聚合的方法合成了基于 1,3,5三乙炔苯的多孔聚合物, 因其在水相中具有良好分散性 的特点, 能够有效光催化降解罗丹明 B 有机染料.

研究人员通常使用端炔基和卤素的 Sonogashira 偶 联反应在较高的温度下制备各种优良性能 PAE 微孔材 料. 近来, 通过金属催化末端炔烃的氧化偶联均聚已经 成为一种制备 PAE 微孔骨架材料的新方法. Zhou 等 ${ }^{[22]}$ 通过四(4-乙炔基苯基)甲烷的偶联均聚合得到聚合物 PPN-1, 其 BET 比表面积为 $1249 \mathrm{~m}^{2} \cdot \mathrm{g}^{-1}$, 在 $1 \mathrm{bar} / 77 \mathrm{~K}$ 条件下 $\mathrm{H}_{2}$ 吸附能力为 $1.37 \mathrm{wt} \%$. 随后, 他们 ${ }^{[43]}$ 以四㶧基 联苯为单体通过偶联均聚得到 PPN-10, BET 比表面积为 $1128 \mathrm{~m}^{2} \cdot \mathrm{g}^{-1}$. Guo 等 ${ }^{[44]}$ 采用室温端炔基氧化均聚的方法, 将四炔基四苯基锌卟啉在细乳液体系中聚合成 CMPs 微 球, 可以实现微球的分散液对于 $\mathrm{SO}_{2}$ 气体的变色检测.
以上研究结果表明通过改变含炔单体分子的大小、长度 和结构类型等, 可以有效地调控制备的有机微孔材料的 比表面积、孔径分布等孔隙率参数以及实现在气体吸 附、环境催化和荧光检测等领域的应用.

相较于其他类型的 MOPs 而言, 对于目前报道的绝 大部分由端炔基单体氧化均聚所得到的 PAE 微孔骨架 材料, 其 $\mathrm{CO}_{2}$ 的捕获能力及 $\mathrm{CO}_{2}$ 选择性分离性能均相对 较低. 研究表明, 向微孔材料骨架中引入 $\mathrm{N}, \mathrm{Si}, \mathrm{O}$ 等原 子可以有效提高与气体的相互作用位点, 从而提高 $\mathrm{CO}_{2}$ 吸附性能 ${ }^{[4,46]}$.

在本工作中我们分别选用三(4-乙炔基)苯胺、甲基 三(4-乙炔基苯基)硅烷、苯基三(4-乙炔基苯基)硅烷为单 体, 通过端炔基氧化均聚的方法, 制备了三种 PAE 微孔 骨架材料, 合成路线如 Scheme 1 所示. 研究结果表明, 制备的微孔骨架材料具有较好的化学稳定性、热稳定性 和适中的 BET 比表面积. 三(4-乙炔基)苯胺聚合得到的 聚合物(TEPA-MOP)的比表面积在制备三种 MOPs 中最 大 $\left(715 \mathrm{~m}^{2} \cdot \mathrm{g}^{-1}\right)$. 在 $1.13 \mathrm{bar} / 273 \mathrm{~K}$ 条件下, TEPA-MOP 的 $\mathrm{CO}_{2}$ 吸附能力为 $1.59 \mathrm{mmol} \cdot \mathrm{g}^{-1}$. 此外, 三种 $\mathrm{MOPs}$ 均展 现良好的 $\mathrm{CO}_{2} / \mathrm{N}_{2}$ 选择性吸附, 其中 TEPP-MOP 的 $\mathrm{CO}_{2} / \mathrm{N}_{2}$ 选择性吸附数值高达 73.2.
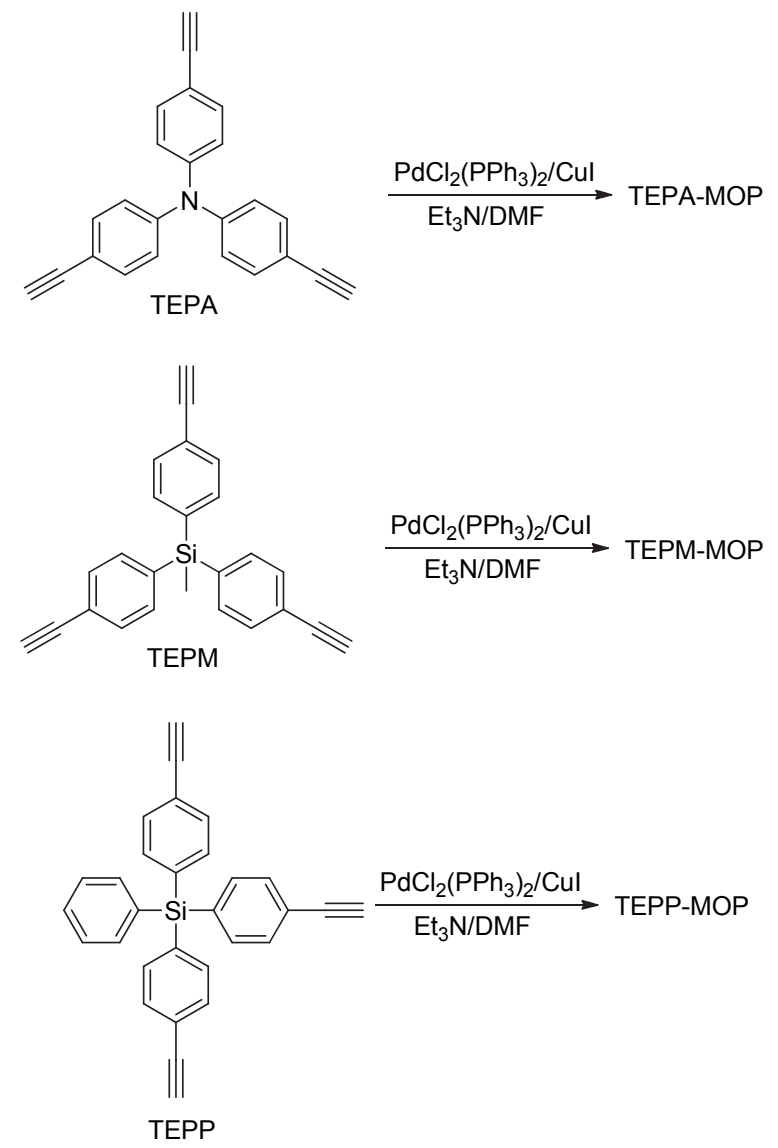

图式 1 聚合物的合成路线

Scheme 1 Synthetic routes for the polymer frameworks 


\section{2 结果与讨论}

\section{1 聚合物的合成与表征}

如 Scheme 1 所示, 分别使用三(4-乙炔基)苯胺、甲 基三(4-乙炔基苯基)硅烷、苯基三(4-乙炔基苯基)硅烷为 单体, Pd(II)与碘化亚铜作催化剂, 三乙胺与二甲基甲酰 胺 (DMF) 作溶剂, 通过氧化均聚反应制得聚合物 TEPA-MOP, TEPM-MOP 和 TEPP-MOP.

测定了单体和氧化均聚反应制得的 PAE 微孔聚合 物的红外光谱图(图 S1). 从单体的红外谱图中可以明确 观察到位于 $3280 \mathrm{~cm}^{-1}$ 的端炔基 $\alpha$ 位 $\mathrm{C}-\mathrm{H}$ 键伸缩振动 峰. 在制备聚合物的红外光谱中, 其特征峰完全消失, 表明在聚合反应过程中单体的端炔基官能团反应的很 彻底 ${ }^{[41]}$.

我们通过热重分析仪来测定所制备聚合物的热稳 定性能(图 S2), 在 $\mathrm{N}_{2}$ 气氛下, 制备的聚合物对应于 $5 \%$ 热失重的热分解温度在 $350{ }^{\circ} \mathrm{C}$ 左右. 当温度达到 $800{ }^{\circ} \mathrm{C}$ 时, 三种聚合物的重量损失比例约为 $20 \%$, 说明 所得微孔聚合物的热稳定性较好. 除此之外, 三种聚合 物具有良好的化学稳定性, 能够在甲醇、三氯甲烷、丙 酮及四氢呋喃等常用有机溶剂中稳定存在. 微孔聚合物 具有上述良好的热稳定性和化学稳定性的主要原因在 于它们自身结构的高度交联性 ${ }^{[27]}$.

为了详细了解聚合物的微观形貌和内部结构, 我们
对聚合物分别进行了扫描电子显微镜(SEM)和透射电子 显微镜图(TEM)的表征. 如图 1 所示, 所有的聚合物呈 现出絮状交织孔道. 从图中也可以看到聚合物存在少量 的大孔，这主要是聚合物分子间由于无序的堆积形成的 孔隙. TEM 测试证明聚合物材料具有蠕虫状的孔结构 (图 S3). 从聚合物粉末的 X 射线衍射谱图(图 S4)中可以 看出聚合物呈现出较宽的衍射峰, 进一步证明聚合物本 质上属于无定形材料.

\section{2 聚合物孔道性能}

我们根据 $77 \mathrm{~K}$ 下的 $\mathrm{N}_{2}$ 的吸附和脱附等温线分析制 备聚合物的比表面积和孔道结构. 如图 2a 所示, 三种聚 合物均表现为 $\mathrm{I}$ 型 $\mathrm{N}_{2}$ 吸附特性. 当相对压力处于低压区 内时, $\mathrm{N}_{2}$ 吸附量迅速增加, 说明在所合成的聚合物中存 在许多的微孔结构. 当相对压力大于 0.8 时, 气体吸附量 仍稍许增加, 这可能是由于样品中颗粒间堆积形成的介 孔和大孔所致. 从图中 $\mathrm{N}_{2}$ 吸附-脱附等温线均可观察到 回滞环的存在, 主要是由于微孔聚合物尤其是无序的纳 米微孔材料的柔性骨架易发生自身弹性形变与膨胀, 狭 窄的孔道导致气体进出孔受阻, 从而导致滞留现象 ${ }^{[41,47]}$. 三种聚合物的孔道性质详见表 S1, 聚合物 TEPA-MOP 的 BET 比表面积最大, 约为 $715 \mathrm{~m}^{2} \cdot \mathrm{g}^{-1}$. TEPM-MOP 和 TEPP-MOP 的比表面积很接近, 分别为 602 和 616 $\mathrm{m}^{2} \cdot \mathrm{g}^{-1}$. 我们用非局部密度泛函理论(NLDFT)计算聚合

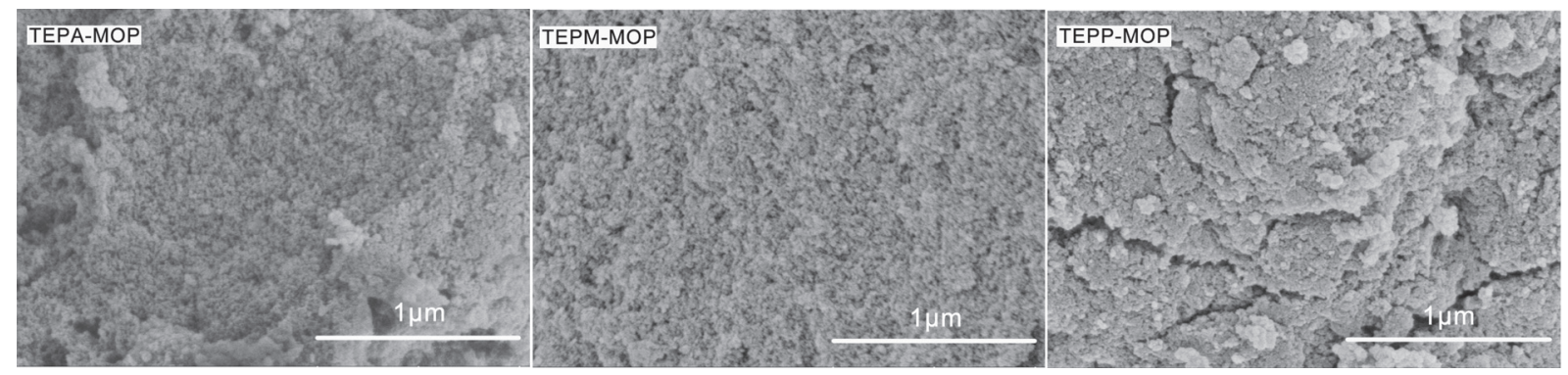

图 1 聚合物的扫描电镜照片

Figure 1 SEM images for the polymer frameworks
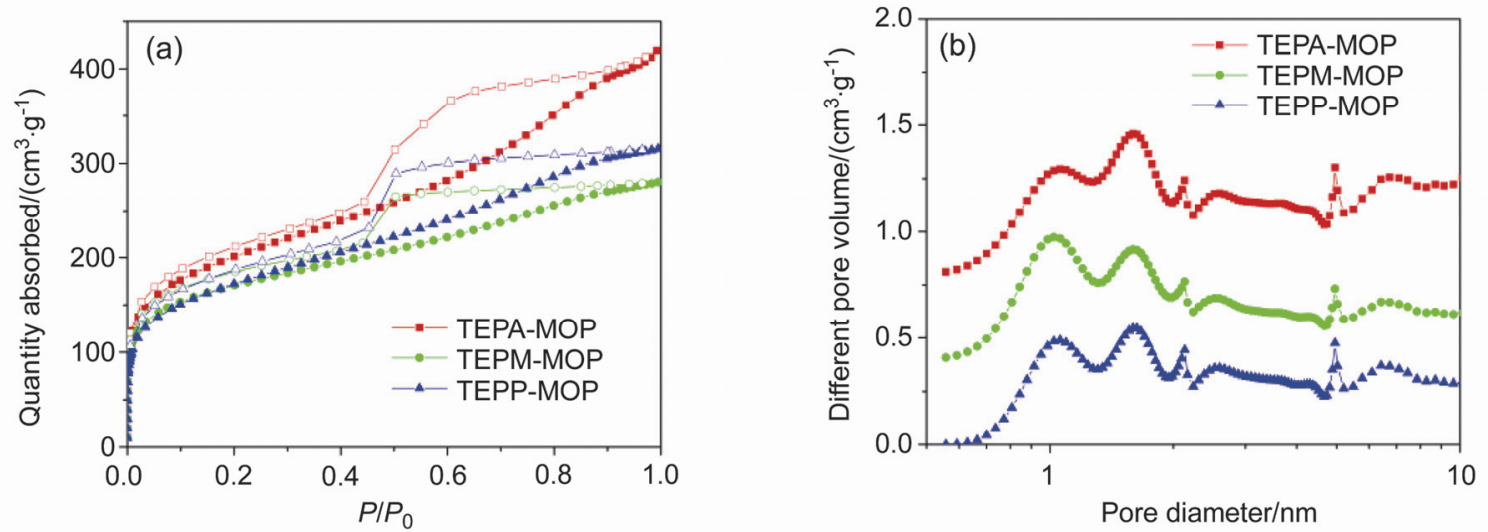

图 2 聚合物的氮气吸附-脱附等温曲线(a)及孔径分布曲线(b)

Figure $2 \mathrm{~N}_{2}$ adsorption (filled symbols)-desorption (empty symbols) isotherms (a) and the pore size distribution curves (b) for the polymer frameworks 
物的孔径分布(图 2b). 如图 2b 所示, 三种聚合物主要微 孔孔径约为 1.1 与 $1.6 \mathrm{~nm}$, 此外还存在少量孔径约为 2.1 与 $4.9 \mathrm{~nm}$ 的介孔结构, 表明聚合物的孔尺寸较窄且分布 主要集中在微孔区域, 是标准的有机微孔材料.

\section{3 聚合物气体吸附性能}

微孔聚合物具有较高的比表面积、较窄的孔径分布, 与小分子气体可能存在较强的相互作用. 此外, 聚合物 TEPA-MOP 中高电荷密度的 $\mathrm{N}$ 原子位点可能提高骨架 的电子传导率增强聚合物骨架与客体分子间的偶极-偶 极相互作用, 从而提高聚合物的气体吸附性能 ${ }^{[45]}$. 基于 这些原因, 我们研究聚合物对 $\mathrm{CO}_{2}, \mathrm{H}_{2}$ 和 $\mathrm{CH}_{4}$ 的吸附作 用, 其测试结果如表 1 所示. 图 $3 \mathrm{a}$ 与图 $3 \mathrm{~b}$ 分别为聚合 物在 273 及 $298 \mathrm{~K}$ 下的 $\mathrm{CO}_{2}$ 吸附曲线. 在 $273 \mathrm{~K} / 1.13 \mathrm{bar}$ 条件下, 聚合物 TEPA-MOP, TEPM-MOP 和 TEPP-MOP
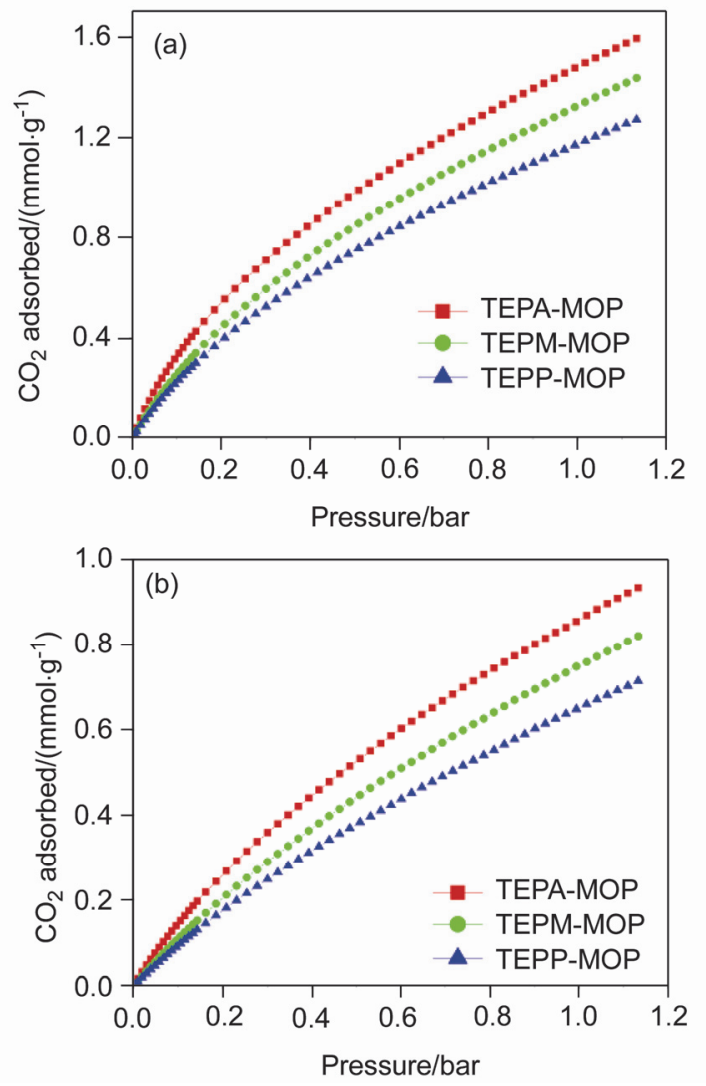

图 3 聚合物在 $273 \mathrm{~K}$ (a)、298 K (b)下的 $\mathrm{CO}_{2}$ 吸附曲线

Figure $3 \mathrm{CO}_{2}$ adsorption curves for the polymer frameworks at $273 \mathrm{~K}$ (a) and (b) $298 \mathrm{~K}$
对 $\mathrm{CO}_{2}$ 的吸附量分别是 $1.59,1.43$ 和 $1.27 \mathrm{mmol} \bullet \mathrm{g}^{-1}$. TEPA-MOP 具有更高的 $\mathrm{CO}_{2}$ 的吸附量, 可能由于在其聚 合物微孔骨架结构中 $\mathrm{N}$ 原子位点上有更高的电荷密度, 因此增强了与 $\mathrm{CO}_{2}$ 的偶极相互作用. 此外, 相比 TEPM-MOP 和 TEPP-MOP, TEPA-MOP 具有较高的比表 面积, 有助于提高对 $\mathrm{CO}_{2}$ 的吸附能力. TEPA-MOP 对 $\mathrm{CO}_{2}$ 的吸附量在相同条件下 $(273 \mathrm{~K} / 1.13 \mathrm{bar})$ 接近部分报 道的同类型共轭微孔 PAE 聚合物, 如聚三(4-乙炔苯)胺



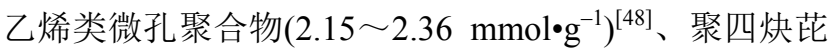
基微孔聚合物 $\left(0.90 \sim 1.16 \mathrm{mmol}^{\circ} \mathrm{g}^{-1}\right)^{[34]}$ 、聚三炔三苯基

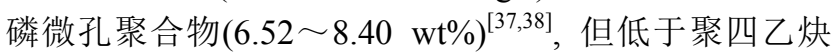
苯基甲烷类微孔聚合物 $\left(2.11 \sim 5.06 \mathrm{mmol} \cdot \mathrm{g}^{-1}\right)^{[26,29,30]}$.

为了进一步了解聚合物与 $\mathrm{CO}_{2}$ 的作用, 我们基于聚 合物在 273 及 $298 \mathrm{~K}$ 下 $\mathrm{CO}_{2}$ 吸附数据, 利用 ClausiusClapeyron 方程公式计算了它们的 $\mathrm{CO}_{2}$ 吸附热. 如图 4 所示, 聚合物 TEPA-MOP, TEPM-MOP 和 TEPP-MOP 的 $\mathrm{CO}_{2}$ 等比容吸附热分别是 $26.4,25.6$ 和 $27.8 \mathrm{~kJ} \cdot \mathrm{mol}^{-1}$. 这 三种聚合物的 $\mathrm{CO}_{2}$ 吸附热与类似结构 PAE 微孔骨架材 料的吸附热大小相当.

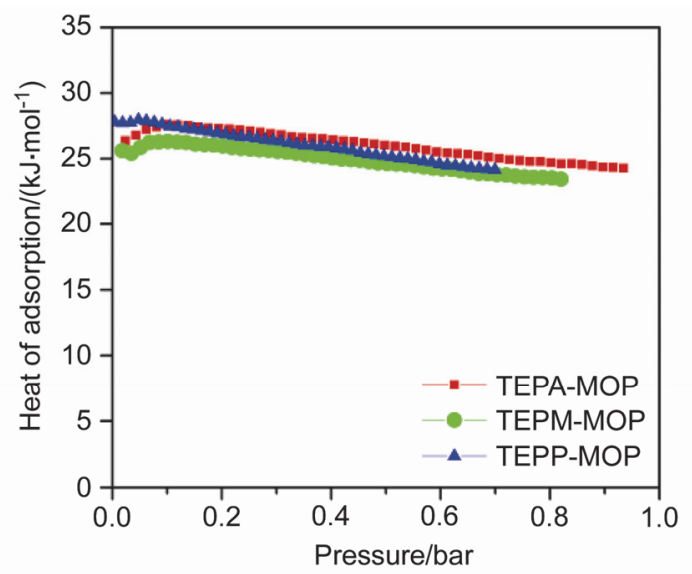

图 4 聚合物对 $\mathrm{CO}_{2}$ 的吸附热

Figure 4 Isosteric heats of adsorption for $\mathrm{CO}_{2}$

$\mathrm{H}_{2}$ 和 $\mathrm{CH}_{4}$ 是一类清洁能源, 在新能源汽车和燃料电 池等领域具有潜在的应用前景，我们进一步研究聚合物 对 $\mathrm{H}_{2}$ 和 $\mathrm{CH}_{4}$ 的吸附作用. 图 $5 \mathrm{a}$ 为聚合物在 $77.3 \mathrm{~K}$ 和绝 对压力至 1.13 bar 条件下测得的 $\mathrm{H}_{2}$ 吸附等温曲线, 聚合 物 TEPA-MOP 表现出最强吸附能力 $1.04 \mathrm{wt} \%$, 而在同

表 1 气体吸附及分离指数

Table 1 Gas uptake and selectivity

\begin{tabular}{|c|c|c|c|c|c|}
\hline \multirow{2}{*}{ Polymer } & \multirow{2}{*}{$\mathrm{H}_{2}$ uptake $^{a} / \mathrm{wt}^{\mathrm{O}} \%$} & \multirow{2}{*}{$\mathrm{CH}_{4}$ uptake $^{b} /\left(\mathrm{mmol} \cdot \mathrm{g}^{-1}\right)$} & \multirow{2}{*}{$\mathrm{CO}_{2}$ uptake $^{b} /\left(\mathrm{mmol} \cdot \mathrm{g}^{-1}\right)$} & \multicolumn{2}{|c|}{ Henry law selectivity ${ }^{c}$} \\
\hline & & & & $\mathrm{CO}_{2} / \mathrm{N}_{2}$ & $\mathrm{CO}_{2} / \mathrm{CH}_{4}$ \\
\hline TEPA-MOP & 1.04 & 0.48 & 1.59 & 69.9 & 5.0 \\
\hline TEPM-MOP & 0.79 & 0.42 & 1.43 & 32.3 & 5.3 \\
\hline TEPP-MOP & 0.64 & 0.39 & 1.27 & 73.2 & 4.6 \\
\hline
\end{tabular}

${ }^{a}$ Data were obtained at 1.13 bar and $77.3 \mathrm{~K} ;{ }^{b}$ Data were obtained at 1.13 bar and $273 \mathrm{~K}^{c}{ }^{c}$ Adsorption selectivity based on the Henry's law. 
样条件下聚合物 TEPM-MOP 与 TEPP-MOP 的吸附性能 只有 $0.79 \mathrm{wt} \%$ 和 $0.64 \mathrm{wt} \%$. TEPA-MOP 的 $\mathrm{H}_{2}$ 吸附量明显 高于 TEPM-MOP 与 TEPP-MOP, 主要是因为聚合物 TEPA-MOP 具有更高的比表面积, 而在 $\mathrm{H}_{2}$ 吸附中起主 要作用的是微孔结构. TEPA-MOP 的 $\mathrm{H}_{2}$ 吸附量接近报道 的其它 PAE 微孔骨架材料, 例如基于 1,3,5-三乙炔基苯

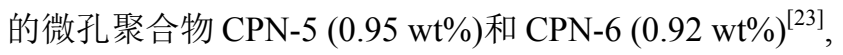
基于四(4-乙炔基苯基)甲烷均聚合所得的聚合物 PPN-1 $(1.37 \mathrm{wt} \%)^{[42]}$. 由于聚合物 TEPA-MOP 具有较大的比表 面积, 同样表现出较好的 $\mathrm{CH}_{4}$ 吸附性能. 如图 5b 所示, 在 $273 \mathrm{~K} / 1.13$ bar 条件下, TEPA-MOP 对 $\mathrm{CH}_{4}$ 的吸附量是

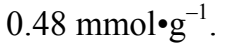


图 5 (a)在 $77.3 \mathrm{~K}$ 温度下的 $\mathrm{H}_{2}$ 吸附曲线和(b)在 $273 \mathrm{~K}$ 温度下的 $\mathrm{CH}_{4}$ 吸附曲线

Figure 5 (a) $\mathrm{H}_{2}$ adsorption curves for the polymer frameworks at $77.3 \mathrm{~K}$ and (b) $\mathrm{CH}_{4}$ adsorption isotherms collected at $273 \mathrm{~K}$

为了研究聚合物的气体选择性吸附, 在相同条件下 通过容量法测量 $\mathrm{CO}_{2}, \mathrm{CH}_{4}$ 和 $\mathrm{N}_{2}$ 的吸附性能, 在低压 $\left(P / P_{0}<0.15 \mathrm{bar}\right)$ 条件下，气体的选择性吸附是根据原始 数据利用 Henry 定律常数的比率计算的单组分气体吸附 等温线的斜率估算得到(图 S5 S7). 如表 1 所示, 聚合 物 TEPA-MOP, TEPM-MOP 和 TEPP-MOP 对 $\mathrm{CO}_{2} / \mathrm{N}_{2}$ 的 选择性吸附分别是 69.9, 32.3 和 73.2. TEPP-MOP 表现出 最低的 $\mathrm{CO}_{2}$ 吸附量和较小的比表面积, 但是 TEPP-MOP 的 $\mathrm{CO}_{2} / \mathrm{N}_{2}$ 选择性吸附却是最高的. 这可能是因为
TEPP-MOP 具有较大的微孔比例 ${ }^{[49,50]}$. 此外, 相比 TEPA-MOP 和 TEPM-MOP, 聚合物 TEPP-MOP 侧链上 苯基官能团的体积大，孔道内空间位阻大，不利于 $\mathrm{N}_{2}$ 分 子的吸收, 导致选择性 $\left(\mathrm{CO}_{2} / \mathrm{N}_{2}\right)$ 提高. 虽然本工作中聚 合物 TEPA-MOP 和 TEPP-MOP 的 $\mathrm{CO}_{2} / \mathrm{N}_{2}$ 的选择性吸附 性能低于文献中报道的选择性吸附大于 100 的有机微孔 聚合物性能, 如四苯基乙烯类聚合物 (Network-7, $119)^{[51]}$, 偶氮类纳米微孔聚合物(azo-COP-2, 110 $)^{[52]}$, 富 含电子的微孔共价框架结构(PAF-1，109) ${ }^{[53]}$; 但与同类 型 PAE 微孔骨架材料相比处于优良水平，如基于四(4乙炔苯基)甲烷微孔聚合物(PAF-18，8.4 25; PAF-35,

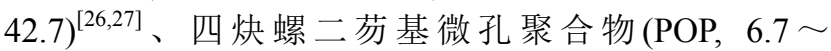
$11.8)^{[36]}$ 、基于四(4-乙炔苯基)硅烷微孔聚合物(20.5 $64.7)^{[41]}$. 需要指出的是, 在很多文献报道中, $\mathrm{CO}_{2}$ 吸附 与理想的 $\mathrm{CO}_{2} / \mathrm{N}_{2}$ 选择吸附性之间可能具有较明显的对 立关系, 也就是说, 部分具有很高 $\mathrm{CO}_{2}$ 吸附能力的微孔 聚合物通常具有较低的选择性. 因此, 聚合物 TEPA-MOP 具有适中的 $\mathrm{CO}_{2}$ 吸附和良好的 $\mathrm{CO}_{2} / \mathrm{N}_{2}$ 选择 性吸附使它在 $\mathrm{CO}_{2}$ 捕获或者封存技术具有应用潜力.

我们进一步研究聚合物的 $\mathrm{CO}_{2} / \mathrm{CH}_{4}$ 选择性吸附性 能. 如表 1 所示, 聚合物的 $\mathrm{CO}_{2} / \mathrm{CH}_{4}$ 选择性吸附在 5.0 左右. 该数值与报道过的大部分 PAE 有机微孔聚合物的 $\mathrm{CO}_{2} / \mathrm{CH}_{4}$ 选择性吸附是相当的 ${ }^{[29,36,37,41,42]}$.

\section{3 结论}

通过端炔基氧化均聚的方法设计合成了三种三臂 连接结构的 PAE 微孔骨架材料. 聚合物在酸、碱及有机 溶剂中都能稳定存在, 并且表现出良好的热稳定性. $\mathrm{N}_{2}$ 吸附结果表明，聚合物比表面积的范围在 $602 \sim 715$ $\mathrm{m}^{2} \cdot \mathrm{g}^{-1}$. 由于在聚合物骨架中引入的 $\mathrm{N}$ 原子能够增强聚 合物孔结构与 $\mathrm{CO}_{2}$ 分子间的作用力, TEPA-MOP 的 $\mathrm{CO}_{2}$ 吸附能力在 $273 \mathrm{~K} / 1.13 \mathrm{bar}$ 条件下为 $1.59 \mathrm{mmol} \cdot \mathrm{g}^{-1}$, 性 能优于其它两种聚合物(TEPM-MOP 和 TEPP-MOP). 此 外, TEPA-MOP 和 TEPP-MOP 具有优良的选择性吸附性 能，对 $\mathrm{CO}_{2} / \mathrm{N}_{2}$ 的选择性吸附分别是 69.9 和 73.2.

虽然本工作中制备的 PAE 微孔骨架材料表现出优 异的选择性吸附性能，但是相比其它类型的有机微孔聚 合物, 聚合物 TEPA-MOP, TEPM-MOP 和 TEPP-MOP 都 存在 $\mathrm{CO}_{2}$ 吸附能力较低的缺点. 相信在不久的将来, 更 多通过端炔基氧化均聚方法制备的具有优异性能的 PAE 微孔骨架材料将被开发和应用，基于 PAE 有机微孔 材料的研究会对材料科学和其它领域的发展起到深远 的影响.

\section{4 实验部分}

\section{1 单体的合成}

本工作中三种单体三(4-乙炔基)苯胺(TEPA)、甲基 三(4-乙炔基苯基)硅烷(TEPM)、苯基三(4-乙炔基苯基) 
硅烷(TEPP)的合成步骤和表征见支持信息(图 S8 S10).

\section{2 聚合物的合成}

以 TEPA-MOP 的合成为例, 将三(4-乙炔基)苯胺 (200 mg, $0.63 \mathrm{mmol}), \mathrm{PdCl}_{2}\left(\mathrm{PPh}_{3}\right)_{2}$ (15 mg, $\left.0.5 \mathrm{mmol}\right)$, $\mathrm{CuI}(10 \mathrm{mg})$ 加入单口烧瓶中, 再用 $\mathrm{N}_{2}$ 对装置进行三次 换气, 分别将三乙胺 $(5 \mathrm{~mL})$ 及 DMF $(5 \mathrm{~mL})$ 打入烧瓶中. 升温至 $100{ }^{\circ} \mathrm{C}$ 并保持匀速搅拌进行反应, $36 \mathrm{~h}$ 后, 依次 用蒸馏水、甲醇、二氯甲烷和丙酮洗涤, 之后得到的聚 合物固体 TEPA-MOP 放在真空烘箱中保持真空干燥 12 h $(182 \mathrm{mg}$, 产率 $92 \%)$.

TEPM-MOP 的合成方法与 TEPA-MOP 相同, 使用 甲基三(4-乙炔基苯基)硅烷(150 mg，0.43 mmol)代替三 (4-乙炔基)苯胺作为原料. 最终产物通过洗涤干燥处理 后产率为 $82 \%$.

TEPP-MOP 的合成方法与 TEPA-MOP 相同, 使用 苯基三(4-乙炔基苯基)硅烷(200 mg，0.49 mmol)代替三 (4-乙炔基)苯胺作为原料. 最终产物通过洗涤干燥处理 后产率为 $87 \%$.

\section{References}

[1] Monastersky, R. Nature 2013, 497, 13

[2] Wu, Z. K.; Huang, Z. L.; Zhang, Y.; Qin, Y. H.; Ma, J. Y.; Luo, Y. B. Chem. Eng. J. 2016, 295, 64

[3] Wang, T. L.; Jens, K. J. Int. J. Greenh. Gas Con. 2015, 37, 354.

[4] Luo, C.; Zheng, Y.; Guo, J.; Feng, B. Fuel 2014, 127, 124.

[5] Dawson, R.; Cooper, A. I.; Adams, D. J. Prog. Polym. Sci. 2012, 37, 530.

[6] Tan, L. X.; Tan, B. Chem. Soc. Rev. 2017, DOI: 10.1039/ c6cs00851h.

[7] Das, S.; Heasman, P.; Ben, T.; Qiu, S. L. Chem. Rev. 2017, 117, 1515.

[8] Cooper, A. I. Adv. Mater. 2009, 21, 1291.

[9] Jiang, J. X.; Cooper, A. I. Top. Curr. Chem. 2010. 293, 1.

[10] Xu, Y. H.; Jin, S. B.; Xu, H.; Nagai, A.; Jiang, D. L. Chem. Soc. Rev. 2013, 42, 8012.

[11] Budd, P. M.; Ghanem, B. S.; Makhseed, S.; McKeown, N. B.; Msayib, K. J.; Tattershall, C. E. Chem. Commun. 2004, 230.

[12] McKeown, N. B.; Budd, P. M. Macromolecules 2010, 43, 5163.

[13] Côté, A. P.; Benin, A. I.; Ockwig, N. W.; O'Keeffe, M.; Matzger, A. J.; Yaghi, O. M. Science 2005, 310, 1166.

[14] Ding, S. Y.; Wang, W. Chem. Soc. Rev. 2013, 42, 548.

[15] Feng, X.; Ding, X. S.; Jiang, D. L. Chem. Soc. Rev. 2012, 41, 6010.

[16] Tan, L. X.; Tan, B. Acta Chim. Sinica 2015, 73, 530. (谭良骁，谭必 恩, 化学学报, 2015, 73, 530.)

[17] Xu, S. J.; Luo, Y. L.; Tan, B. Macromol. Rapid. Commun. 2013, 34, 471.

[18] Luo, Y.; Li, B.; Wang, W.; Wu, K.; Tan, B. Adv. Mater. 2012, 24, 5703.

[19] Ren, S. J.; Bojdys, M. J.; Dawson, R.; Laybourn, A.; Khimyak, Y. Z.; Adams, D. J.; Cooper, A. I. Adv. Mater. 2012, 24, 2357.

[20] Ben, T.; Ren, H.; Ma, S. Q.; Cao, D. P.; Lan, J. H.; Jing, X. F.; Wang, W. C.; Xu, J.; Deng, F.; Simmons, J. M.; Qiu, S. L.; Zhu, G. S. Angew. Chem., Int. Ed. 2009, 48, 9457.

[21] Yuan, Y.; Yan, Z. J.; Ren, H.; Liu, Q. Y.; Zhu, G. S.; Sun, F. X. Acta Chim. Sinica 2012, 70, 1446. (元野, 间卓君, 任浩, 刘青英, 朱广 山, 孙福兴, 化学学报, 2012, 70, 1446.)
[22] Jiang, J. X.; Su, F. B.; Trewin, A.; Wood, C. D.; Campbell, N. L.; Niu, H. J.; Dickinson, C.; Ganin, A. Y.; Rosseinsky, M. J.; Khimyak, Y. Z.; Cooper, A. I. Angew. Chem., Int. Ed. 2007, 46, 8574.

[23] Jiang, J. X.; Su, F. B.; Trewin, A.; Wood, C. D.; Niu, H. J.; Jones, J. T.; Khimyak, Y. Z.; Cooper, A. I. J. Am. Chem. Soc. 2008, 130, 7710 .

[24] Jiang, J. X.; Su, F. B.; Niu, H. J.; Wood, C. D.; Campbell, N. L.; Khimyak, Y. Z.; Cooper, A. I. Chem. Commun. 2008, 486.

[25] Jiang, J. X.; Trewin, A.; Su, F. B.; Wood, C. D.; Niu, H. J.; Jones, J. T. A.; Khimyak, Y. Z.; Cooper, A. I. Macromolecules 2009, 42, 2658.

[26] Ma, H. P.; Ren, H.; Zou, X. Q.; Sun, F. X.; Yan, Z. J.; Cai, K.; Wang, D. Y.; Zhu, G. S. J. Mater. Chem. A 2013, 1, 752.

[27] Yuan, R. R.; Ren, H.; Yan, Z. J.; Wang, A. F.; Zhu, G. S. Polym Chem. 2014, 5, 2266

[28] Yan, Z. J.; Yuan, Y.; Tian, Y. Y.; Zhang, D. M.; Zhu, G. S. Angew. Chem., Int. Ed. 2015, 54, 12733.

[29] Ma, H. P.; Ren, H.; Zou, X. Q.; Meng, S.; Sun, F. X.; Zhu, G. S. Polym. Chem. 2014, 5, 144.

[30] Yang, Z. Z.; Zhao, Y. F.; Zhang, H. Y.; Yu, B.; Ma, Z. S.; Ji, G. P.; Liu, Z. M. Chem. Commun. 2014, 50, 13910.

[31] Thompson, C. M.; McCandless, G. T.; Wijenayake, S. N.; Alfarawati, O.; Jahangiri, M.; Kokash, A.; Tran, Z.; Smaldone, R. A. Macromolecules 2014, 47, 8645 .

[32] Thompson, C. M.; Li, F.; Smaldone, R. A. Chem. Commun. 2014 50,6171

[33] Trunk, M.; Herrmann, A.; Bildirir, H.; Yassin, A.; Schmidt, J.; Thomas, A. Chem. Eur. J. 2016, 22, 7179.

[34] Yan, Z. J.; Ren, H.; Ma, H. P.; Yuan, R. R.; Yuan, Y.; Zou, X. Q.; Sun, F. X.; Zhu, G. S. Microporous Mesoporous Mater. 2013, 173, 92.

[35] Chen, Q.; Wang, J. X.; Wang, Q.; Bian, N.; Li, Z. H.; Yan, C. G.; Han, B. H. Macromolecules 2011, 44, 7987.

[36] Ma, Q. Y.; Yang, B. X.; Li, J. Q. RSC Adv. 2015, 5, 64163.

[37] Qiao, S. L.; Du, Z. K.; Yang, C. P.; Zhou, Y. H.; Zhu, D. Q.; Wang, J. X.; Chen, X. H.; Yang, R. Q. Polymer 2014, 55, 1177.

[38] Qiao, S. L.; Huang, W.; Du, Z. K.; Chen, X. H.; Shieh, F. K.; Yang, R. Q. New J. Chem. 2014, 39, 136.

[39] Ma, B. C.; Ghasimi, S.; Landfester, K.; Vilela, F.; Zhang, K. A. I. J. Mater. Chem. A 2015, 3, 16064.

[40] Zhang, T. T.; Wang, H. T.; Ma, H. P.; Sun, F. X.; Cui, X. Q.; Zhu, G. S. Acta Chim. Sinica 2013, 71, 1598. (张婷婷, 王海涛, 马和平, 孙福兴, 崔小强, 朱广山, 化学学报, 2013, 71, 1598.)

[41] Zhang, H. J.; Zhang, C.; Wang, X. C.; Qiu, Z. X.; Liang, X. M.; Chen, B.; Xu, J. W.; Jiang, J. X.; Li, Y. D.; Li, H.; Wang, F. RSC Adv. 2016, 6, 113826.

[42] Lu, W. G.; Yuan, D. Q.; Zhao, D.; Schilling, C. I.; Plietzsch, O.; Muller, T.; Brase, S.; Guenther, J.; Blumel, J.; Krishna, R.; Li, Z.; Zhou, H. C. Chem. Mater. 2010, 22, 5964.

[43] Lu, W. G.; Wei, Z. W.; Yuan, D. Q.; Tian, J.; Fordham, S.; Zhou, H. C. Chem. Mater. 2014, 26, 4589.

[44] Wu, K. Y.; Guo, J.; Wang, C. C. Chem. Commun. 2014, 50, 695.

[45] Cao, Q.; Chen, Q.; Han, B. H. Acta Chim. Sinica 2015, 73, 541. (操 强, 陈琦, 韩宝航, 化学学报, 2015, 73, 541.)

[46] Zhang, Y. H.; Li, Y. D.; Wang, F.; Zhao, Y.; Zhang, C.; Wang, X. Y.; Jiang, J. X. Polymer 2014, 55, 5746.

[47] Zhang, C.; Kong, R.; Wang, X.; Xu, Y. F.; Wang, F.; Ren, W. F.; Wang, Y. H.; Su, F. B.; Jiang, J. X. Carbon 2017, 114, 608.

[48] Zhao, Y.; Wang, X. Y.; Zhang, C.; Jiang, J. X. Acta Chim. Sinica 2015, 73, 634. (赵洋, 王笑颜, 张崇, 蒋加兴, 化学学报, 2015, 73, 634.)

[49] Zhang, X.; Lu, J. Z.; Zhang, J. Chem. Mater. 2014, 26, 4023.

[50] Dawson, R.; Cooper, A. I.; Adams, D. J. Polym. Int. 2013, 62, 345.

[51] Yao, S. W.; Yang, X.; Yu, M.; Zhang, Y. H.; Jiang, J. X. J. Mater Chem. A 2014, 2, 8054 .

[52] Patel, H. A.; Je, S. H.; Park, J.; Jung, Y.; Coskun, A.; Yavuz, C. T. Chem. Eur. J. 2014, 20, 772.

[53] Mohanty, P.; Kull, L. D.; Landskron, K. Nat. Commun. 2011, 2, 401.

(Zhao, C.) 